\title{
An interpretable machine learning model for diagnosis of Alzheimer's disease
}

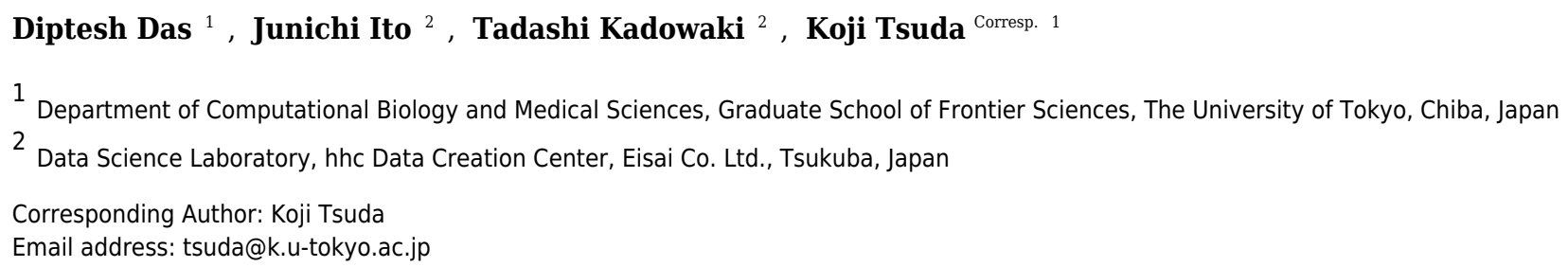

We present an interpretable machine learning model for medical diagnosis called SHIMR (sparse high-order interaction model with rejection option). A decision tree explains to a patient the diagnosis with a long rule (i.e., conjunction of many intervals), while SHIMR employs a weighted sum of short rules. Using proteomics data of 151 subjects in the Alzheimer's Disease Neuroimaging Initiative (ADNI) dataset, SHIMR is shown to be as accurate as other non-interpretable methods (Sensitivity, $S N=0.84 \pm 0.1$, Specificity, $\mathrm{SP}=0.69 \pm 0.15$ and Area under the curve, $\mathrm{AUC}=0.86 \pm 0.09)$. For clinical usage, SHIMR has a function to abstain from making any diagnosis when it is not confident enough, so that a medical doctor can choose more accurate but invasive and/or more costly pathologies. Incorporation of rejection option complements SHIMR to design a multistage cost-effective diagnosis framework. Using baseline concentration of cerebrospinal fluid (CSF) and plasma proteins of 141 common cohort, SHIMR is shown to be effective to design a patient specific cost-effective Alzheimer's disease (AD) pathology. Thus, interpretability, reliability and having the potential to design a patient specific multistage cost-effective diagnosis framework can make SHIMR serve as an indispensable tool in the era of precision medicine that can cater to the demand of both doctor and patients, and reduce the overwhelming financial burden of medical diagnosis. 


\section{An interpretable machine learning model for diagnosis of Alzheimer's disease}

\section{Diptesh Das $^{1}$, Junichi Ito ${ }^{2}$, Tadashi Kadowaki ${ }^{2}$, and Koji Tsuda ${ }^{1, \dagger}$}

\author{
${ }^{1}$ Department of Computational Biology and Medical Sciences, Graduate School of \\ Frontier Sciences, The University of Tokyo, Chiba 277-8561, Japan \\ 2Data Science Laboratory, hhc Data Creation Center, Eisai Co. Ltd., 5-1-3 Tokodai, \\ Tsukuba 300-2635, Japan
}

\author{
Corresponding author: \\ Koji Tsuda ${ }^{\dagger}$ \\ Email address: tsuda@k.u-tokyo.ac.jp
}

\section{INTRODUCTION}

Alzheimer's disease (AD) is a progressive disease affecting memory and other mental functionalities with deteriorating symptoms over time. With increased human life expectancy, a large number $(11 \sim 16$ million) of elderly people are likely to suffer from AD by 2050 (Alzheimer's, 2015). Treatment of AD is often hampered due to the lack of easily accessible and cost-effective biomarkers with reliable diagnostic accuracy. To counter this problem, Alzheimer's Disease Neuroimaging Initiative (ADNI) study began in 2004 with the intention of collecting and storing multitude of data spanning across clinical, imaging, omics, gene expression data etc. Fluid based biomarkers e.g. cerebrospinal fluid (CSF) and neuroimaging such as magnetic resonance imaging (MRI) or positron emission tomography (PET) are highly accurate, but often not feasible for clinical implementation due to either high cost or invasive in nature or lack of specialized clinics offering such services. Consequently, effective treatments are only available to limited patients. These limitations have significant impact on both the patients lacking effective treatment for $\mathrm{AD}$ as well as the health care system trying to cope with substantial financial burden (Henriksen et al., 2014). Therefore, the goal of ADNI core research (Henriksen et al., 2014) is to find a cost-effective way (e.g. blood based biomarkers or cognitive assessment) that can serve as the first step in a multistage diagnostic or prognostic process followed by most advanced and expensive pathologies such as CSF or MRI screening. Another important aspect is that it is not feasible for a medical practitioner, expert in this domain, to exploit such a vast and diverse datasets manually. Hence, there exists an urgent need of advanced computer-aided diagnosis (CAD) framework that can serve as a helping hand to a medical practitioner to better understand the disease and design a patient specific medical regime. A line of 
research has been conducted to device such CAD methods. Deep learning based automated diagnosis framework (Fisher et al. (2018); Lu et al. (2018); Charan et al. (2018)), hyper spectral imaging based methods (Khan et al. (2018)) are examples of state of the art CAD methods. However, it is often the case that a medical practitioner cannot rely on state-of-the-art CAD methods despite its high accuracy. Because, most of these are opaque and cannot answer the basic question- why/how has it reached to such a decision and why/how is it biologically relevant? (Freitas et al., 2010; Freitas, 2006; Burrell, 2016; Ribeiro et al., 2016b). Recently European Union has issued a "General Data Protection Regulation (GDPR)" on algorithmic decision-making and a "right to explanation" (Goodman and Flaxman, 2016) which mandate that a data subject has the right to "meaningful information about the logic involved in the decision making". In other words, the GDPR requires that communication with data subjects has to be made in a "concise, intelligible, and easily accessible form". Therefore, to cater to the demand of both, a medical practitioner (doctor) and a subject (patient), the most effective approach would be the design of a cost-effective multistage CAD framework where the trained model can be articulated and understood by a human. In a sense the model should provide enough information about how input features relate to predictions and allow one to answer questions such as: Is the prediction biologically relevant?, Which features play the most important role in prediction? Why am I diagnosed as diseased/normal? or Why the prescribed treatment is the optimum one given my current medical condition?. For these reasons, existing CAD methods involving rule induction algorithms such as propositional rule, decision table, decision tree etc.(Huysmans et al., 2011; Kim et al., 2014; Wang and Rudin, 2015) are often preferred with the aim of generating an interpretable set of 'if-then' rules. A line of research (Ribeiro et al., 2016c,a; Zhou et al., 2003) has also been conducted to extract set of human understandable rules from black box models (neural networks, support vector machines etc.). However, previous research(Huysmans et al., 2006; Johansson et al., 2005) on rule induction algorithms and posthoc rule extraction methods either suffer from very complex rule generation or result in a suboptimal set of decision rules owing to the nature of optimization problem formulated in their computational model. Another longstanding concern of a domain expert to embrace a rule based computation model is its poor representation, as highlighted by Pazzani (Pazzani, 2000) that a very little effort has been invested to empirically assess interpretabilty beyond simply reporting the size of the resulting representations. Pazzani (Pazzani, 2000) also highlighted that there have been no attempt of visual representation, whereas users prefer certain visualization over mere textual or graphical description. To address the above issues, we present a "Sparse High order Interaction Model with Rejection option" (SHIMR).

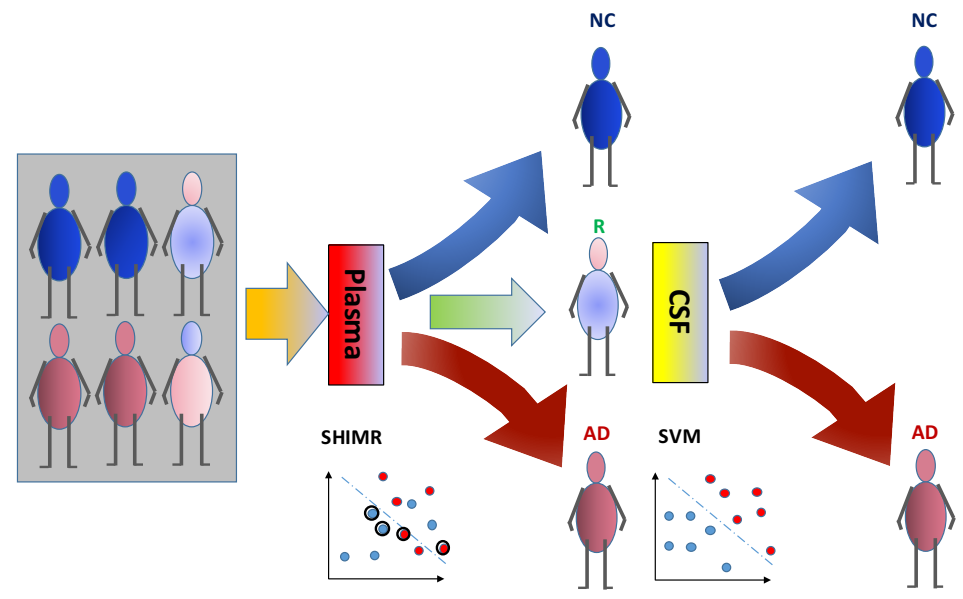

Figure 1. Cost-effective multistage framework for the diagnosis of Alzheimer's disease (AD) patients from normal control (NC). In a clinical setting, all registered patients can undergo an initial screening using inexpensive and easily accessible biomarkers (e.g. Plasma). Only those patients difficult to diagnosis (hence "Rejected: R" by SHIMR) are recommended for invasive and/or more expensive screening (e.g. CSF). Abbreviation: CSF, cerebrospinal fluid; SVM, support vector machines. 
A) SHIMR Decision Rule:

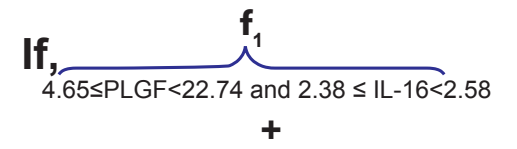

$\underbrace{1.73 \leq A P O E<1.98 \text { and } 2.20 \leq S G O T<3.6 \text { and } 3.28 \leq B N P \leq 3.76}_{\text {then, "AD" }}$

C)

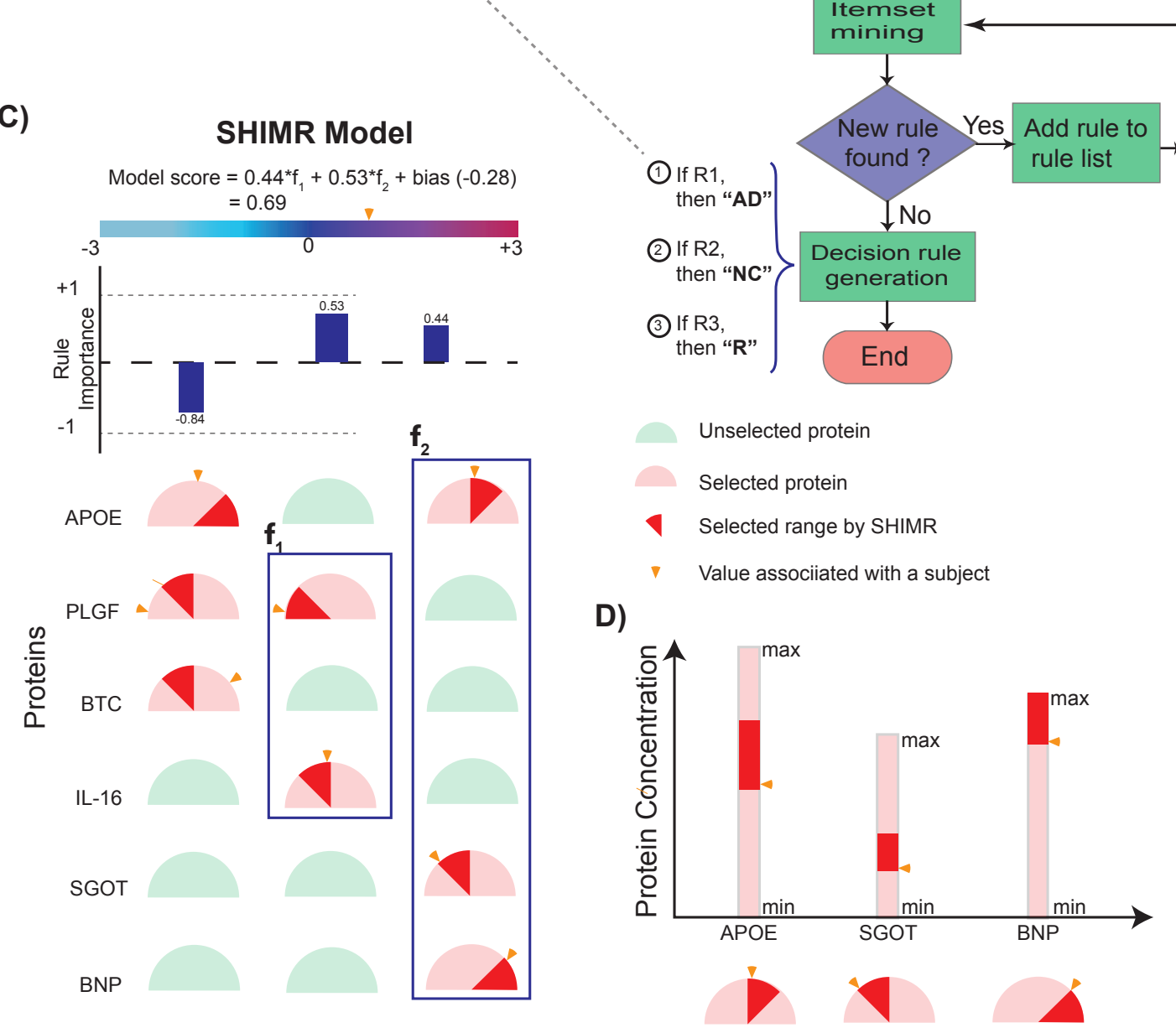

B) SHIMR Flow Diagram:

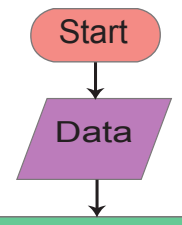

Binarize data to generate rules

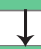

Itemset mining

Figure 2. Visualization of SHIMR. The selected rules (A), which are generated by SHIMR (B), are described as an intersection matrix (C). Each row in the intersection matrix (C) represents individual protein and each column represents interaction among proteins constituting a rule. Proteins selected by a rule are represented by 'light red gauge' (semicircles), whereas the unselected ones by 'light green gauge'. The exact selected range of a particular protein is highlighted by 'dark red wedge'. The blue rectangular box surrounding a set of proteins highlights the selected protein combination (or feature) for a subject. Blue colored bars above each column show the importance of each rule contributing to the overall "model score". The generated model score for a subject (patient) is also highlighted by an 'orange pointer' over a color bar at the top of each plot. This color bar describes the overall range of model scores. Construction of the intersection matrix from different concentrations of individual proteins is also shown (D). Each gauge represents the concentration range of a particular protein where the left end represents the minimum and the right end represents the maximum. The 'orange pointer' over each protein gauge describes the exact value of protein concentration corresponding to a particular subject. Abbreviation: $\mathrm{NC}$, normal control; AD, Alzheimer's disease, R, rejected; R1, rule 1; R2, rule 2; R3, rule 3; f1, feature 1 of rule $1 ;$ f2, feature 2 of rule 1 . 
The incorporation of rejection option complements SHIMR to design a multistage cost-effective framework (Fig. 1) with the notion of refraining from making any decision (Reject them: R) for those data patients which are hard to classify (patients who are close to the decision boundary of a CAD model) and make prediction for only those patients for which the model is confident enough (patients which are far apart from decision boundary of a CAD model). Patients rejected due to the low confidence of first stage model, trained on inexpensive and easily accessible biomarkers (e.g. plasma) can further be recommended for second stage of evaluation, involving advanced and complex screening (e.g. CSF). However, a medical practitioners must be able to interpret the model and identify the variables separately for rejected and classified samples and decide the subsequent course of treatment accordingly. To address that, SHIMR employs interval conjunction rules as highly interpretable as well as accurate decision sets. Decision sets which consist of sets of 'if-then' rules are in general simple, concise and highly interpretable (Lakkaraju et al., 2016) as shown in Fig. 2 generated by our visualization module. Another potential advantage of using interval conjunction rules is that it can capture the combinatorial interactions of multiple factors which can prove to be beneficial to decipher complex clinical phenomenon such as Alzheimer's disease which is otherwise difficult to explain using single biomarker as highlighted by Henriksen et al. (Henriksen et al., 2014).

To validate our model, we considered diagnostic classification of Alzheimer's disease (AD) patients from normal control (NC) using ADNI dataset (http://adni.loni.usc.edu/). Experimental results show that our method can generate highly interpretable as well as accurate machine learning model which can serve as an indispensable tool in the era of precision medicine. By considering a hypothetical cost model, we have also shown how our method can lead to a cost-effective diagnosis framework. A python implementation of SHIMR can be downloaded from https://github.com/tsudalab/ SHIMR.

\section{METHODS}

Data. Data used in the preparation of this article were obtained from the Alzheimer's Disease Neuroimaging Initiative (ADNI) database (adni.loni.usc.edu). The ADNI was launched in 2003 as a public-private partnership, led by Principal Investigator Michael W. Weiner, MD. The primary goal of ADNI has been to test whether serial magnetic resonance imaging (MRI), positron emission tomography (PET), other biological markers, and clinical and neuropsychological assessment can be combined to measure the progression of mild cognitive impairment (MCI) and early AD. The plasma protein data was obtained from 'Biomarkers Consortium Plasma Proteomics Project RBM multiplex data', which contains 190 proteins previously reported in the literature to be related to human pathogenesis. Other data (such as demographic, diagnosis, MMSE and UPENN CSF biomarkers) were collected from ADNIMERGE R package (ADNIMERGE_0.0.1.tar.gz). All these data were downloaded from ADNI web-site (http: //adni.loni.usc.edu/) as of Mar. 23, 2016.

Model. First, we briefly review existing machine learning based approaches and highlight their limitation to generate an interpretable CAD model which motivated the design of SHIMR. Kernel based methods such as support vector machines (SVM) have been widely used for last two decades in several machine learning applications (Schölkopf and Smola, 2002). In SVM, the decision function is defined as $f(x)=$ $\sum_{i=1}^{n} a_{i} K\left(x, x_{i}\right)$. where, $K$ is a called the kernel function which is in general a non-linear function (Gaussian, polynomial kernels etc.) used to measure the similarity between any training example $x$ and a support vector $x_{i}$. Neural networks (Haykin, 2001) have similar decision functions using different non-linear activation functions (logistic, tanh etc.). Due to such non-linear transformation it is often possible to generate highly accurate learning model but at the expense of losing model interpretability a.k.a. "black box" model. To this end, decision trees are often used which are generally interpretable as well as highly accurate(Beerenwinkel et al., 2002). The 'if-then-else' rules employ Boolean clauses with logical AND and NOT operators $(\wedge, \neg)$ to constitute complex features. However, complex features in decision trees have limited forms and boosting is often combined (Dietterich, 2000) to circumvent such limitations, but at the cost of losing interpretability. To address this limitation, SHIMR represents the decision function as a weighted sum of conjunction rules, each of which is the conjunction of one-dimensional intervals. A conjuction rule looks like $I\left(1.0 \leq x_{3} \leq 2.0\right) I\left(x_{5} \geq 0.5\right) I\left(x_{6} \leq 0.8\right)$, where $I(\cdot)$ refers to the indicator function.

The model generated by SHIMR consists of sets of independent 'if-then' rules which are in general 
A)

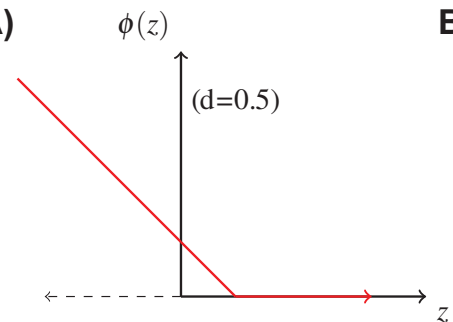

$$
f(x)=\sum_{j=1}^{M} a_{j} h_{j}(x)+b .
$$

simple, concise and highly interpretable compared to decision list (Lakkaraju et al., 2016). This simple stucture allows intuitive visualization shown in Fig. 2. Complexity of decision list (Blum, 1998; Klivans and Servedio, 2006; Valiant, 1999; Clark and Niblett, 1989) comes from the 'if-then-else' clause of rule formation. Because of the 'else' conjugate one needs to consider all the preceding rules that have already been turned out to be false to make a decision. SHIMR's closest ancestor is itemset boosting (Saigo et al., 2007), but it has extended functionalities for medical diagnosis such as dealing with continuous attributes, rejection option, class imbalance (Veropoulos et al., 1999), calibration and visualization.

Let $\mathbb{H}=\left\{h_{1}, \ldots, h_{M}\right\}$ denote the set of all possible conjunction rules, where each feature $x_{i}$ is divided into a fixed number of intervals. SHIMR learns the following decision function from data,

where $x$ is the feature vector of a patient, $a_{j}$ is a weight associated with $h_{j}$, and $b$ is the bias term. In learning from data $\left\{x_{i}, y_{i}\right\}_{i=1}^{n}$, the following objective function is minimized with respect to $a_{j}$ and $\mathrm{b}$.

$$
\sum_{j=1}^{M}\left|a_{j}\right|+C^{+} \sum_{\left\{i \mid y_{i}=1\right\}} \phi\left(f\left(x_{i}\right)\right)+C^{-} \sum_{\left\{i \mid y_{i}=-1\right\}} \phi\left(-f\left(x_{i}\right)\right)
$$

where $\phi$ is a loss function explained in the next section, $C^{+}$and $C^{-}$are the regularization parameters for positive and negative classes, respectively. This is an extremely high dimensional problem, but at the optimal solution, there are only a limited number of non-zero weights due to L1-norm regularization. We employ the column generation method (Demiriz et al., 2002) that starts from the optimization problem with no variables and gradually grows the problem by adding one variable in each iteration. For selecting a variable efficiently, weighted itemset mining (Uno et al., 2005) is used. Refer to "Supplemental Methods" of Article $\mathrm{S} 1$ for details about the learning procedure.

Decision making from $f(x)$ is affected by the cost of rejection. Rejection is not so bad as misclassifiation, but incurs some cost as we need a different means for final decision. Assuming that the cost of misclassification is one, let us define $0 \leq d \leq 0.5$ as the cost of rejection. Bartlett and Wegkamp (Bartlett and Wegkamp, 2008) showed that, if $\eta(x)=P(y=1 \mid x)$ is the posterior probability of $x$ being classified to the positive class, the following decision rule achieves the smallest expected cost,

$$
f_{d}^{*}(x)=\left\{\begin{array}{rl}
+1 & \eta(x)>1-d \\
0 & d \leq \eta(x) \leq 1-d \\
-1 & \eta(x)<d
\end{array}\right.
$$

We use the above rule after converting $f(x)$ to the posterior probability via isotonic calibration.

Figure 3. Double hinge loss function at different values of rejection cost $d$.

Loss function If the cost of rejection is known, it is reasonable to incorporate it in the loss function $\phi$ in the learning procedure. We use the following double hinge function proposed by Bartlett and Wegkamp (Bartlett and Wegkamp, 2008),

$$
\phi(z)= \begin{cases}0 & z \geq 1 \\ 1-z & 0 \leq z<1 \\ 1-\frac{(1-d) z}{d} & z<0\end{cases}
$$

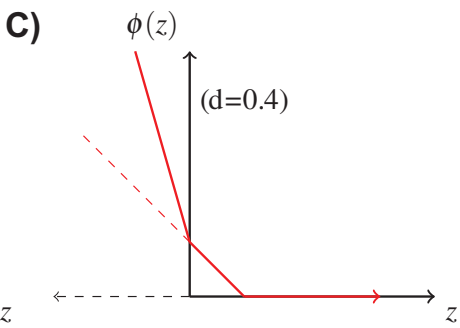



sensitivity (SN) and specificity (SP).

\begin{tabular}{|c|c|c|c|c|c|}
\hline Diagnosis & \# of Subjects & Age & Gender (M/F) & Education & MMSE \\
\hline AD & 97 & $74.89 \pm 7.97$ & $53 / 44$ & $15.21 \pm 3.08$ & $21.25 \pm 4.62$ \\
\hline NC & 54 & $75.32 \pm 5.84$ & $28 / 26$ & $15.6 \pm 2.82$ & $29.06 \pm 1.21$ \\
\hline
\end{tabular}

Table 1. Demographic information of 151 subjects from ADNI dataset used in this work. Abbreviations: AD, Alzheimer's disease; NC, normal control; MMSE, Mini mental state examination. Note: Standard deviations of variables Age, Education and MMSE scores are shown after ' \pm ' sign. of $d$. When $d=0.5$, it is equivalent to the normal hinge loss. As $d$ decreases, the slope in the negative domain becomes steeper, because the cost of misclassification becomes higher in comparison to $d$.

\section{RESULTS}

\section{Experimental Settings}

In this study we considered baseline concentration of plasma proteins of 151 subjects, out of which 97 subjects were diagnosed as AD patients and the remaining 54 subjects as NC. Here, we considered 14 proteins as the starting set of analytes. This is a collection of proteins responsible for AD pathology as reported in literature (Llano, D.A. et al. Signature \#4 (Llano et al., 2013)). The entire list of these 14 plasma proteins can be found in Table S1. We also considered baseline concentration of cerebrospinal fluid (CSF) and plasma proteins of 141 common cohort to demonstrate a cost-effective diagnosis framework. Out of these 141 subjects, 88 subjects were diagnosed as AD and 53 subjects were diagnosed as NC. For CSF data we used tau, amyloid- $\beta$ (A $\beta$ ) and phosphorylated tau (p-tau) proteins. We mainly used the ratio tau/A $\beta$ and $\mathrm{p}$-tau/A $\beta$ as the features for CSF analysis. Baseline demographic information of all 151 subjects used in the current study is shown in Table 1. The entire dataset is divided into two stratified groups (two-third Train and one-third Test) using the same strategy as reported in Llano, D.A. et al.(Llano et al., 2013). Model generation and hyper parameters selection have been done using only the train dataset, whereas the unseen test data is used to report the classification test performance. To validate the performance of our method we have reported both internal cross validation as well as test results (Llano et al., 2013). All the hyper parameters of the model are selected based on five-fold cross validation by running it ten times. To report the internal cross validation performance, the training data has been divided into five stratified groups. At each iteration, four folds are used to generate the model which is subsequently used to generate the results for the held out fold. This procedure is repeated for ten times and the average results have been reported to minimize the data sampling bias. Classification performance has been evaluated using area under the receiver operating characteristics curve (AUC), accuracy (ACC),

\section{Interpretability vs Accuracy Trade-Off}

In this section we will present that our method has the ability to produce comparable accuracy as other existing non-linear methods without compromising the interpretability of the model. We will evaluate the interpretability of our SHIMR model both visually and quantitatively against another interpretable classification model, decision tree (DT) classifier. We will also present how the interpretability trades off classification accuracy and make a comparative study between SHIMR and DT. Fig. 4A \& 4B compare the performance of our method (SHIMR) against existing methods for AD vs NC classification. It can be observed that SHIMR can generate highly accurate classification model comparable to other existing non-linear models. The AUC of internal cross validation was 0.86 with a high sensitivity of 0.84 and reasonable specificity of 0.69 . Next we will show how our visualization module complements SHIMR by generating a simple and easily comprehensible visual representation of the model generated by SHIMR. Our visualization module can clearly represent the weighted combination of simple rules based classification model generated by SHIMR.

Where, $z=\sum_{i=1}^{n} y_{i} f\left(x_{i}\right)$ is the classification margin. Fig. 3 shows the above function for different values 


\begin{tabular}{|c|c|c|c|c|c|c|}
\hline \multicolumn{9}{|c|}{ Internal Cross-Validation } \\
\hline \multirow{2}{*}{ Method } & \multicolumn{2}{|c|}{ SN } & \multicolumn{2}{c|}{ SP } & \multicolumn{2}{c|}{ AUC } \\
\cline { 2 - 7 } & Mean & SD & Mean & SD & Mean & SD \\
\hline RF & 0.79 & 0.03 & 0.86 & 0.02 & 0.82 & 0.02 \\
\hline SVM & 0.80 & 0.12 & 0.64 & 0.16 & 0.79 & 0.08 \\
\hline DT & 0.82 & 0.10 & 0.64 & 0.17 & 0.73 & 0.09 \\
\hline SHIMR & 0.84 & 0.1 & 0.69 & 0.15 & 0.86 & 0.09 \\
\hline
\end{tabular}

B)

\begin{tabular}{|c|c|c|c|}
\hline \multicolumn{4}{|c|}{ External Validation (Test Set) } \\
\hline Method & SN & SP & AUC \\
\hline RF & 0.87 & 0.84 & 0.85 \\
\hline SVM & 0.81 & 0.5 & 0.80 \\
\hline DT & 0.78 & 0.56 & 0.67 \\
\hline SHIMR & 0.84 & 0.67 & 0.81 \\
\hline
\end{tabular}

C)

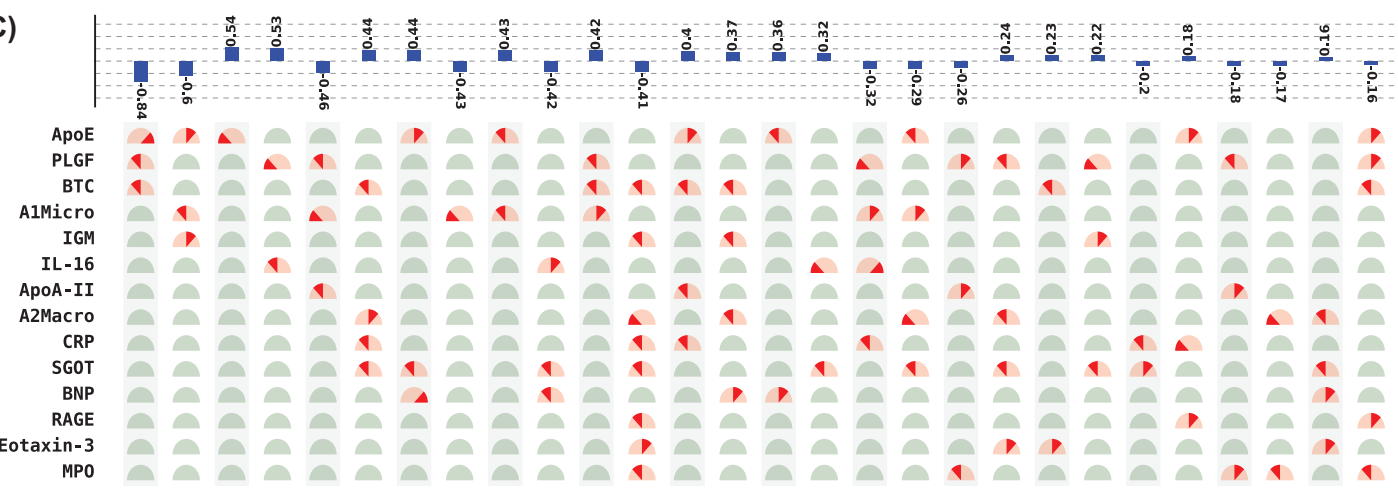

D)

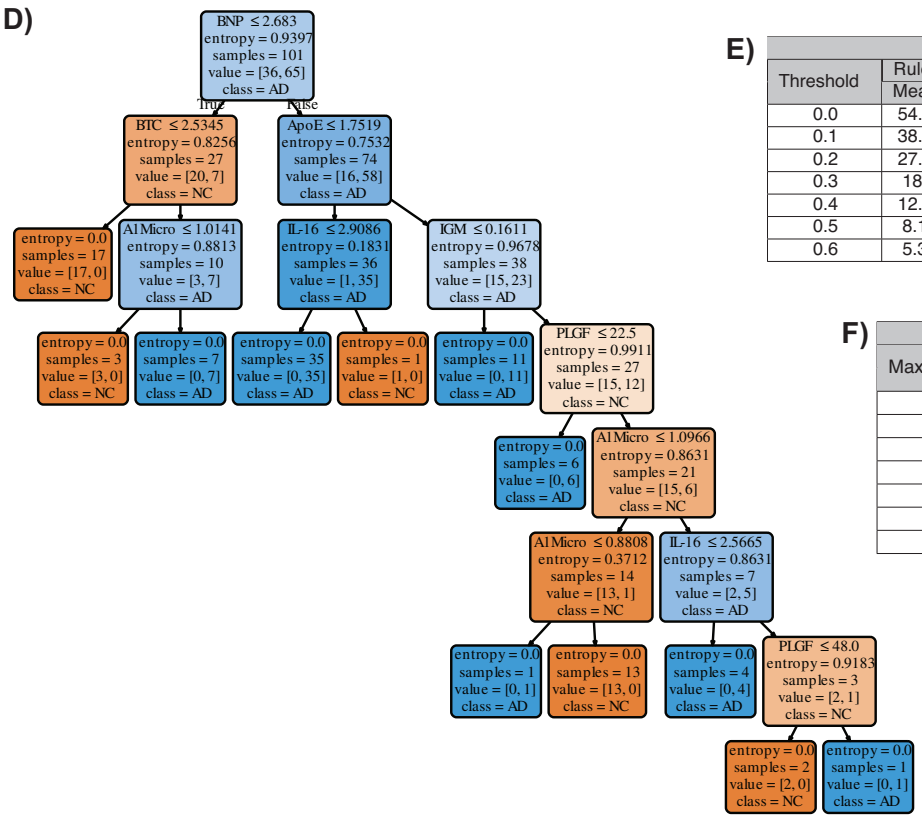

Figure 4. Interpretability vs accuracy trade-off. Comparison of classification (NC vs AD) performance between our method (SHIMR) at zero rejection rate $(R R=0)$ and other standard classifiers (RF, SVM and DT) both in terms of (A) internal cross validation and (B) external test set validation. Visual representation of the model generated by (C) SHIMR and (D) DT respectively. (C) shows the weighted combination of simple rule based classification model generated by SHIMR and (D) shows long chains of conjugated rules generated by DT. Interpretability vs accuracy trade-off comparison between (E) SHIMR and (F) DT. More interpretable model can be generated by tuning the weight threshold of features (in SHIMR) or controlling the maximum depth of tree (in DT) with a compromise in classification accuracy. Abbreviation: RF, random forest; SVM, support vector machines; DT, decision tree; SHIMR, sparse high order interaction model with rejection option; SN, sensitivity; SP, specificity; AUC, area under the curve; SD, standard deviation.

Note: For results against RF, we have quoted the results taken from Llano et al. (2013). In SVM, we introduced nonlinearity by using radial basis function kernel.

In Fig. 4C, the selected rules contributing to the model have been described as an intersection matrix, where each row represents individual feature (protein) and each column represents interaction among features (proteins) constituting a rule. A blue colored bar (above each column) shows the importance 
of each rule contributing to the overall model. Selected features are represented by 'light red gauge' (semicircles), whereas the unselected one by 'light green gauge'. The exact selected range of a particular feature is highlighted using 'dark red wedge'. Looking in the clock-wise direction, each gauge represents the range of a particular feature where the left end represents the minimum and the right end represents the maximum. This representation highlights how interpretable our model is by clearly articulating the trained model in terms of the selected rules, rule importance and the attributes associated with each rule for individual subject. From Fig. 4C, one can understand that the generated rules are simple and easy to understand and possible to validate from domain knowledge. Whereas, DT classifier (Fig. 4D) generates a long chain of conjugated rules for the same classification task. Comparing Fig. 4A \& 4B, one can see that SHIMR can also produce a better classification AUC $(=0.86)$ compared to DT (AUC $=0.73)$. To investigate how interpretability trades off the classification accuracy we further experimented with the feature importance threshold and maximum tree depth in SHIMR and DT respectively, and the corresponding results are shown in Fig. 4E \& 4F. From the results one can observe that a more interpretable model can be generated at the expense of classification accuracy. However, in case of SHIMR, it is possible to generate highly interpretable model (rule length 18 ) at a reasonable classification accuracy ( $\mathrm{AUC}=0.79$ ) which is better than the accuracy ( $\mathrm{AUC}=0.73$ ) of a full (not truncated) decision tree. To generate the full model as shown in Fig. 4C, SHIMR took 17.5 - $18 \mathrm{sec}$ on average, using standard MacBookPro laptop with Intel core i5, $2.9 \mathrm{GHz}$ processor and $8 \mathrm{~GB}$ RAM.

\section{Interpretability vs accuracy trade-off: Comparing SHIMR with CORELS using plasma data.}

CORELS (Angelino et al. (2017)) is a branch-and-bound optimization algorithm for finding optimal rule list from categorical data. CORELS leverages a number of theoretical bounds such as hierarchical objective lower bound, antecedent support lower bound, prefix length upper bound etc. to obtain optimal rule list more efficiently (both in terms of time and space requirements) than existing CART and other decision tree methods. We also compared the classification performance of SHIMR with that of CORELS using ADNI plasma data. Comparing the results of SHIMR (Table S5) with CORELS (Table S3, Table S4), it can be observed that CORELS can produce more interpretable model by generating less number of rules, but at the expense of losing classification accuracy. Accuracy of SHIMR (acc $=0.79$ ) is much higher than the best achieved accuracy $(\mathrm{acc}=0.69$, lambda $=0.02$ ) obtained by CORELS. The details of parameter settings of CORELS and how it has been executed to generate the results of Table S3 and Table S4 can be found in "Supplemental Results" of Article S1.

\section{DISCUSSION}

\section{Adherence to the EU's GDPR on Algorithmic Decision Making and "Right to Explanation".}

The visual representation of SHIMR can also help to explain a specific medical condition and its associated treatment in the context of precision medicine as evident from Fig. 5A \& 5B. The blue rectangular box surrounding a set of proteins highlights the selected protein combination (or feature) for a subject. The 'orange colored wedge' over each protein gauge describes the exact value of protein concentration corresponding to a particular subject. The generated model score for each subject is also highlighted by an orange pointer over a color bar at the top of each plot. From these figures one can understand that a high negative model score (-1.12) attributes to NC, whereas a high positive model score (1.10) attributes to $\mathrm{AD}$ and a model score close to zero corresponds to rejected (R) sample. Here, one can also identify the range of features attributed to $\mathrm{AD}, \mathrm{NC}$ or R. Looking at such an interpretable $\mathrm{CAD}$ model, a doctor can understand the reason behind classification (NC or $\mathrm{AD}$ ) as well rejection (R), and hence design a patient specific diagnostic regime subsequently. Such a model is also helpful for a patient to understand his/her current medical condition, and hence, relate to the treatment prescribed by a doctor. Therefore, SHIMR can be considered to adhere to the EU's GDPR on Algorithmic Decision Making and "Right to Explanation" (Goodman and Flaxman, 2016). 
A)
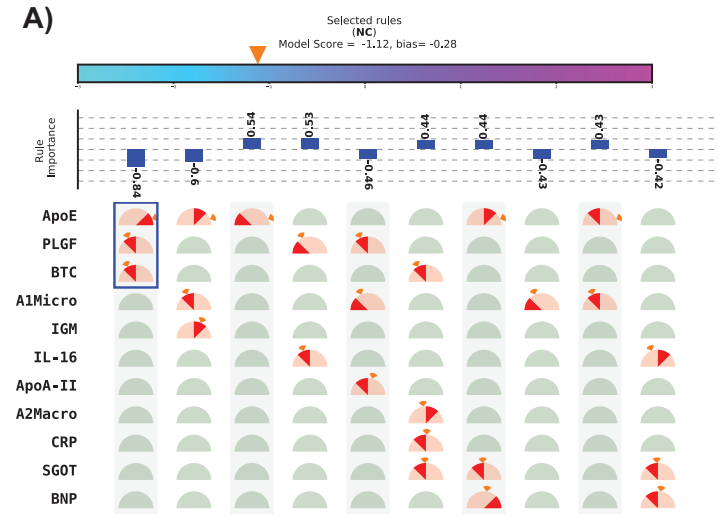

B)
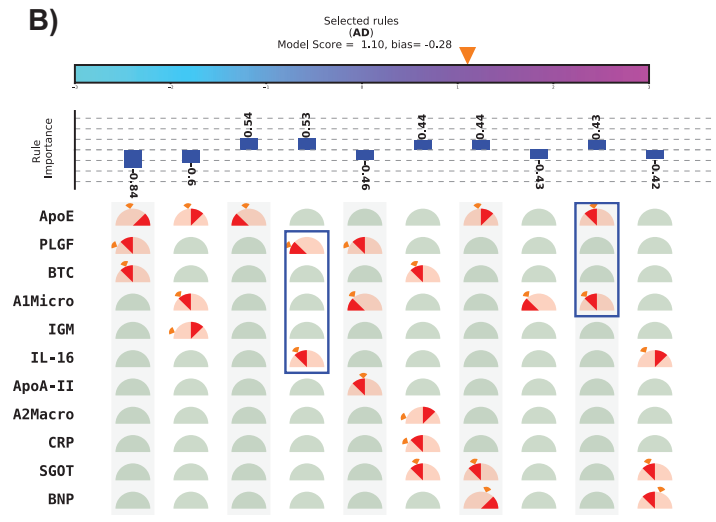

Figure 5. Visual representation of interpretable model generated for individual subject. (A) Normal control (NC) and (B) Alzheimer's disease (AD). The top 10 rules have been depicted in this figure. The model score is generated as the sum of weights of the selected rules and bias. Abbreviations: $\mathrm{NC}$, normal control; AD, Alzheimer's disease.

Note: The bias corresponds to the value of bias term of the model (For details refer to the "Methods" section). Here, we displayed only top 10 rules because of the space constraint. Therefore, sum of the weights of the displayed selected rules may not match the model score as in the case of (B). This will match exactly if the full model is displayed.

\section{Cost-effective Framework}

Treatment of AD is often hampered due to the lack of easily accessible and cost-effective biomarkers with reliable diagnostic accuracy as highlighted in the introduction section. In this section we will evaluate that how our method (SHIMR) can lead to an interpretable cost-effective multistage framework for clinical diagnosis by exploiting the notion of "classification with rejection option". Here we propose a cost-effective framework in the context of precision medicine. Figure 6A describes the effect of rejection for the classification of NC vs AD using plasma and CSF data and how it can be exploited to design a cost-effective pathology for $\mathrm{AD}$ treatment. It can be seen that as the rejection rate is increased, the classification accuracy improves with increased prediction reliability (increased rejection rate infers higher decision threshold). Starting with an ACC of 0.74 at no rejection $(\mathrm{RR}=0)$, it is possible to achieve a classification $\mathrm{ACC}=0.9$ at a higher rejection rate $(\mathrm{RR}=0.38)$ using plasma data (Fig. 6A: Plasma). On the other hand, if CSF data is used for the same classification task, a more reliable prediction (ACC $=0.87$ ) can be achieved with no rejection (Fig. 6A: CSF). Therefore, it can be argued that those 12 subjects ( $R R=0.26$ ) who are rejected using low cost and easily accessible plasma biomarkers can now be recommended for a more sophisticated screening (e.g. CSF biomarkers). It can be observed from Table S2 that 11 out of those 12 subjects can be correctly classified using CSF data. Evidently this highlights the efficacy of CSF screening in the current context. However, as highlighted before, it is not feasible to conduct CSF or other advanced screening for all registered patients due to either invasive in nature, high financial burden or both. To visually depict this trade-off between correctness in diagnosis and cost of screening (Fig. 6B) we assumed a hypothetical cost model for plasma and CSF. Considering accessibility and invasiveness of the screening, we assumed the cost of one CSF screening as 10 unit if the cost of one plasma screening is one unit (10:1 ratio). With that assumption, it can be observed from Fig. 6B that instead of using CSF as the first stage screening which would otherwise cost 470 units for \#of correct classification=41, it would be more cost-effective to consider plasma at the first stage screening followed by CSF screening (cost=167 units). Basically the notion of applying rejection option can help a medical practitioner to decide a systematic clinical regime for each patient individually depending on the confidence of a machine learning model generated based on particular type of data. Hence, it is possible to start from a low cost screening (e.g. plasma) followed by more sophisticated and invasive screening (e.g. CSF) for those patients only for which the model confidence is not so reliable. Hence, effectively controlling the rejection rate for a desired level of AUC (or accuracy) and subsequently applying good source of data, it is possible to design a patient specific cost-effective as well as reliable AD pathology, catering to the need of both patients and health care system (Henriksen et al., 2014). 
A)

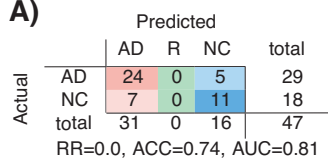

$\mathrm{RR}=0.0, \mathrm{ACC}=0.74, \mathrm{A \cup C}=0.81$

Plasma

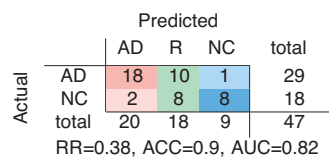

Plasma

C)
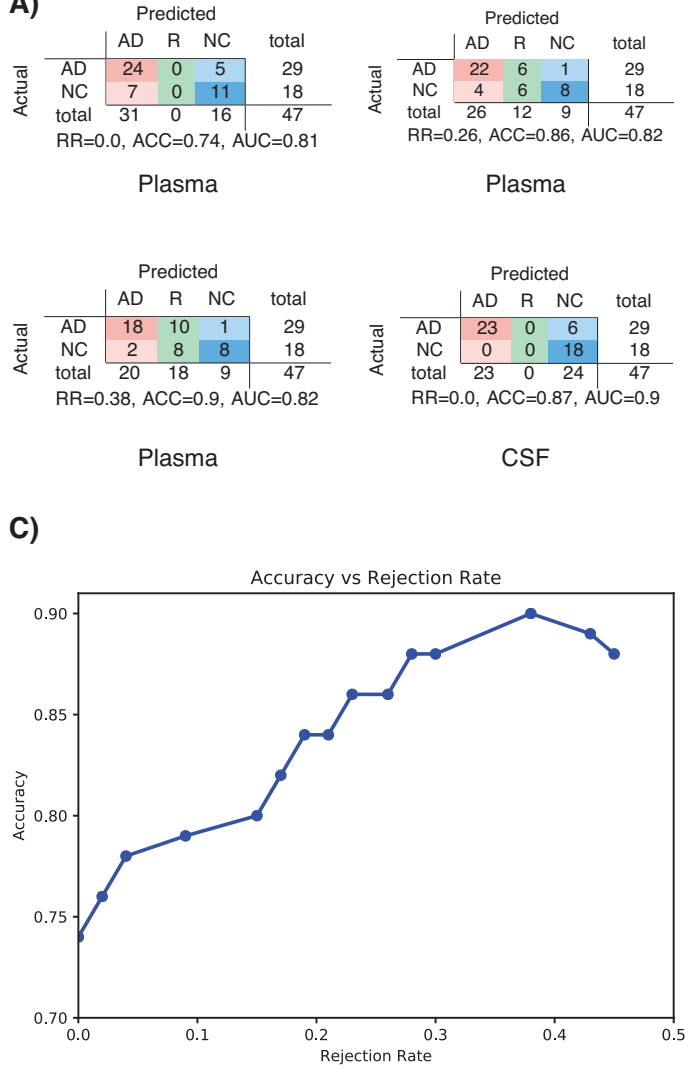

Plasma

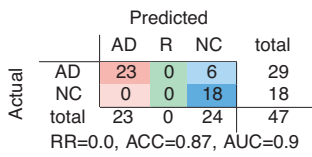

CSF
B)

\begin{tabular}{|c|c|c|c|c|c|}
\cline { 2 - 6 } \multicolumn{1}{c|}{ Total Cost } & 47 & 127 & 167 & 227 & 470 \\
\hline Plasma (cost 1) & 47 & 47 & 47 & 47 & 0 \\
\hline CSF (cost 10) & 0 & 8 & 12 & 18 & 47 \\
\hline
\end{tabular}

D)

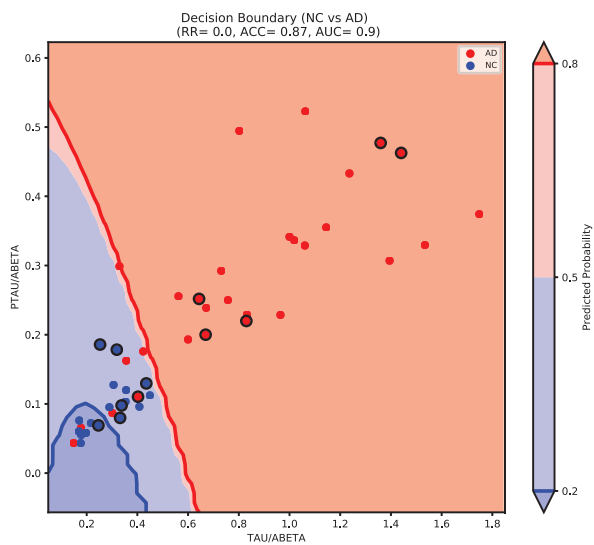

Figure 6. Effect of rejection option to design a cost-effective multistage diagnosis framework. (A) Model performance in terms of confusion matrix, accuracy (ACC) and area under the ROC curve (AUC) at different rejection rates (RR) for Plasma and CSF respectively. (B) Trade-off between correctness of diagnosis and cost of screening. The numerical value below each bar plot refers to the total cost of screening and the numerical value above each bar plot represents the number of correctly diagnosed patients. The table below the bar plot describes the hypothetical cost of individual screening. The table cell entries along each row (Plasma or CSF) represent the cost of individual screening resulting in overall cost as represented by the numerical values below each bar plot. (C) Accuracy vs Rejection Rate trade-off for SHIMR using plasma data. (D) A 2D decision boundary generated by SVM using CSF data. The samples clinically diagnosed as AD or NC are highlighted by red and blue dots respectively. The samples rejected by SHIMR using plasma data are highlighted by drawing a circle around respective dots. Abbreviations: CSF, Cerebrospinal fluid; SVM, support vector machine; SHIMR, sparse high order interaction model with rejection option.

To understand how SHIMR internally works, the accuracy vs rejection rate trade-off for the classification of NC vs AD using plasma data has been depicted in Fig. 6C. It can be observed that as rejection rate is increased, more and more data points which are close to the decision boundary and hence hard to classify get rejected and thus resulted in an improved accuracy after rejection. In order to understand how CSF data can be used to classify those rejected data points, a 2D decision boundary generated by SVM classifier for the same classification task has been plotted (Fig. 6D). The data points rejected by SHIMR using plasma have been highlighted by drawing a circle around respective data points on the same plot. In Fig. 6D, different decision confidence zones based on predicted probabilities have been highlighted using different colors. The dark red region represents high confidence zone for AD with positive predicted probability value more than $80 \%$ and dark blue region represents high confidence zone for NC with positive predicted probability value less than $20 \%$. The region of intermediate positive predicted probability values are highlighted with light shades of respective colors. It is important to mention that SHIMR has the ability to identify the ambiguous low confidence zones (light red or blue) and refrain from taking any decision (reject) for those data points falling in that zone. Therefore, high 
rejection rate conforms to high prediction probability of the classified samples and hence more reliability in prediction. In a sense SHIMR makes decision only for those data points for which it is highly confident (high positive predictive probability) and thus can serve as a highly reliable CAD model to a medical practitioner (e.g. Doctor).

\section{CONCLUSION}

To summarize, we have presented a highly accurate, interpretable and cost effected machine learning framework in the context of precision medicine. We have formulated a sparse high order interaction model with embedded rejection option and solved it using simplex based column generation method. The learning objective function is linear and convex, and hence it is possible to find globally optimum solution. Our method can generate highly accurate and interpretable decision sets which are sets of "if-then" rules capturing the higher order interactions among set of individual features. By embedding rejection option and handling the class imbalance with separate misclassification cost for positive and negative examples, our method can judiciously mange the uncertainty of traditional machine learning based model. To validate the effectiveness of our method, we conducted diagnostic classification of Alzheimer's disease from normal control using ADNI dataset and presented a cost-effective AD pathology. This iterative simultaneous forward feature and sample selection method can lead to a very high accuracy: $\mathrm{ACC}=0.9(\mathrm{AUC}=0.82)$ at $\mathrm{RR}=0.38$ for $\mathrm{AD}$ vs $\mathrm{NC}$ classification using plasma data. This potentially leads to a highly confident prediction model, a much desirable aspect in clinical diagnosis. We have shown that it is possible to design a patient specific systematic multistage cost-effective AD pathology using low cost plasma profile followed by more advanced screening such as CSF. A large scale preventive care is possible by exploiting such patient specific machine learning framework which leverages low cost and easily accessible plasma pathology as an early predictor of $\mathrm{AD}$ and subsequently recommends advanced pathology to those patients only for which it is not possible to generate desired level of accuracy using low cost pathology. However, over-reliance on machine learning based automated diagnosis is a matter of concern as a single "False Negative" is highly expensive as it is associated with the life of the person being treated. Obvious social implication can be who or what would be made responsible for such misdiagnosis. Another concern can be related to the privacy and security of the patient data used for automated diagnosis. Automated diagnosis relies on electronic health records, the very construction of which may induce large and systematic mismeasurement, resulting in bias in automated diagnosis. Interpretable models such as SHIMR alleviates such concerns associated with automated diagnosis, but it does not completely eliminate the role of a medical practitioner in medical diagnosis. A consensus among machine derived diagnosis and diagnosis based on human expert knowledge is desirable. Therefore, human intervention is inescapable in medical diagnosis where a doctor, expert in this domain can validate the automated diagnosis and use CAD as a helping hand and not as an entity of complete reliance.

\section{ACKNOWLEDGEMENTS}

We would like to thank David duVerle and Aika Terada for their fruitful discussions. Data used in preparation of this article were obtained from the Alzheimer's Disease Neuroimaging Initiative (ADNI) database (adni.loni.usc.edu). As such, the investigators within the ADNI contributed to the design and implementation of ADNI and/or provided data but did not participate in analysis or writing of this report. A complete listing of ADNI investigators can be found at: http://adni.loni.usc. edu/wp-content/uploads/how_to_apply/ADNI_Acknowledgement_List.pdf. Data collection and sharing for this project was funded by the Alzheimer's Disease Neuroimaging Initiative (ADNI) (National Institutes of Health Grant U01 AG024904) and DOD ADNI (Department of Defense award number W81XWH-12-2-0012). ADNI is funded by the National Institute on Aging, the National Institute of Biomedical Imaging and Bioengineering, and through generous contributions from the following: AbbVie, Alzheimer's Association; Alzheimer's Drug Discovery Foundation; Araclon Biotech; BioClinica, Inc.; Biogen; Bristol-Myers Squibb Company; CereSpir, Inc.; Cogstate; Eisai Inc.; Elan Pharmaceuticals, Inc.; Eli Lilly and Company; EuroImmun; F. Hoffmann-La Roche Ltd and its affiliated company Genentech, Inc.; Fujirebio; GE Healthcare; IXICO Ltd.; Janssen Alzheimer Immunotherapy Research \& Development, LLC.; Johnson \& Johnson Pharmaceutical Research \& Development LLC.; Lumosity; Lundbeck; Merck \& Co., Inc.; Meso Scale Diagnostics, LLC.; NeuroRx Research; Neurotrack Technologies; Novartis Pharmaceuticals Corporation; Pfizer Inc.; Piramal Imaging; Servier; Takeda 
Pharmaceutical Company; and Transition Therapeutics. The Canadian Institutes of Health Research is providing funds to support ADNI clinical sites in Canada. Private sector contributions are facilitated by the Foundation for the National Institutes of Health (www.fnih.org). The grantee organization is the Northern California Institute for Research and Education, and the study is coordinated by the Alzheimer's Therapeutic Research Institute at the University of Southern California. ADNI data are disseminated by the Laboratory for Neuro Imaging at the University of Southern California.

\section{REFERENCES}

Alzheimer's, A. (2015). 2015 Alzheimer's disease facts and figures. Alzheimer's \& dementia: the journal of the Alzheimer's Association, 11(3):332.

Angelino, E., Larus-Stone, N., Alabi, D., Seltzer, M., and Rudin, C. (2017). Learning certifiably optimal rule lists. In Proceedings of the 23rd ACM SIGKDD International Conference on Knowledge Discovery and Data Mining, pages 35-44. ACM.

Bartlett, P. L. and Wegkamp, M. H. (2008). Classification with a reject option using a hinge loss. Journal of Machine Learning Research, 9(Aug):1823-1840.

Beerenwinkel, N., Schmidt, B., Walter, H., Kaiser, R., Lengauer, T., Hoffmann, D., Korn, K., and Selbig, J. (2002). Diversity and complexity of hiv-1 drug resistance: a bioinformatics approach to predicting phenotype from genotype. Proceedings of the National Academy of Sciences, 99(12):8271-8276.

Blum, A. (1998). On-line algorithms in machine learning. In Online algorithms, pages 306-325. Springer. Burrell, J. (2016). How the machine 'thinks': Understanding opacity in machine learning algorithms. Big Data \& Society, 3(1):2053951715622512.

Charan, S., Khan, M. J., and Khurshid, K. (2018). Breast cancer detection in mammograms using convolutional neural network. In Computing, Mathematics and Engineering Technologies (iCoMET), 2018 International Conference on, pages 1-5. IEEE.

Clark, P. and Niblett, T. (1989). The cn2 induction algorithm. Machine learning, 3(4):261-283.

Demiriz, A., Bennett, K. P., and Shawe-Taylor, J. (2002). Linear programming boosting via column generation. Machine Learning, 46(1):225-254.

Dietterich, T. G. (2000). An experimental comparison of three methods for constructing ensembles of decision trees: Bagging, boosting, and randomization. Machine learning, 40(2):139-157.

Fisher, C. K., Smith, A. M., Walsh, J. R., and the Coalition Against Major Diseases (2018). Deep learning for comprehensive forecasting of alzheimer's disease progression. arXiv preprint arXiv:1807.03876.

Freitas, A. A. (2006). Are we really discovering interesting knowledge from data. Expert Update (the BCS-SGAI magazine), 9(1):41-47.

Freitas, A. A., Wieser, D. C., and Apweiler, R. (2010). On the importance of comprehensible classification models for protein function prediction. IEEE/ACM Transactions on Computational Biology and Bioinformatics (TCBB), 7(1):172-182.

Goodman, B. and Flaxman, S. (2016). European union regulations on algorithmic decision-making and a" right to explanation". arXiv preprint arXiv:1606.08813.

Haykin, S. S. (2001). Neural networks: a comprehensive foundation. Tsinghua University Press.

Henriksen, K., O’Bryant, S. E., Hampel, H., Trojanowski, J. Q., Montine, T. J., Jeromin, A., Blennow, K., Lönneborg, A., Wyss-Coray, T., Soares, H., Bazenet, C., Sjögren, M., Hu, W., Lovestone, S., Karsdal, M. A., Weiner, M. W., and for the Blood-Based Biomarker Interest Group (2014). The future of blood-based biomarkers for Alzheimer's disease. Alzheimer's \& Dementia, 10(1):115-131.

Huysmans, J., Baesens, B., and Vanthienen, J. (2006). Using rule extraction to improve the comprehensibility of predictive models. Fetew research report kbi 0612,technical report, Katholieke Universiteit Leuven.

Huysmans, J., Dejaeger, K., Mues, C., Vanthienen, J., and Baesens, B. (2011). An empirical evaluation of the comprehensibility of decision table, tree and rule based predictive models. Decision Support Systems, 51(1):141-154.

Johansson, U., Konig, R., and Niklasson, L. (2005). Automatically balancing accuracy and comprehensibility in predictive modeling. In Information Fusion, 2005 8th International Conference on, volume 2, pages 7-pp. IEEE.

Khan, M. J., Khan, H. S., Yousaf, A., Khurshid, K., and Abbas, A. (2018). Modern trends in hyperspectral image analysis: A review. IEEE Access, 6:14118-14129.

Kim, B., Rudin, C., and Shah, J. A. (2014). The bayesian case model: A generative approach for 
case-based reasoning and prototype classification. In Advances in Neural Information Processing Systems, pages 1952-1960.

Klivans, A. R. and Servedio, R. A. (2006). Toward attribute efficient learning of decision lists and parities. Journal of Machine Learning Research, 7(Apr):587-602.

Lakkaraju, H., Bach, S. H., and Leskovec, J. (2016). Interpretable decision sets: A joint framework for description and prediction. In Proceedings of the 22Nd ACM SIGKDD International Conference on Knowledge Discovery and Data Mining, KDD '16, pages 1675-1684, New York, NY, USA. ACM.

Llano, D. A., Devanarayan, V., Simon, A. J., and ADNI (2013). Evaluation of plasma proteomic data for Alzheimer disease state classification and for the prediction of progression from mild cognitive impairment to Alzheimer disease. Alzheimer Disease \& Associated Disorders, 27(3):233-243.

Lu, D., Popuri, K., Ding, G. W., Balachandar, R., and Beg, M. F. (2018). Multimodal and multiscale deep neural networks for the early diagnosis of alzheimer's disease using structural $\mathrm{mr}$ and fdg-pet images. Scientific reports, 8(1):5697.

Pazzani, M. J. (2000). Knowledge discovery from data? IEEE intelligent systems and their applications, 15(2):10-12.

Ribeiro, M. T., Singh, S., and Guestrin, C. (2016a). Model-agnostic interpretability of machine learning. arXiv preprint arXiv: 1606.05386.

Ribeiro, M. T., Singh, S., and Guestrin, C. (2016b). Nothing else matters: Model-agnostic explanations by identifying prediction invariance. arXiv preprint arXiv:1611.05817.

Ribeiro, M. T., Singh, S., and Guestrin, C. (2016c). Why should i trust you?: Explaining the predictions of any classifier. In Proceedings of the 22nd ACM SIGKDD International Conference on Knowledge Discovery and Data Mining, pages 1135-1144. ACM.

Saigo, H., Uno, T., and Tsuda, K. (2007). Mining complex genotypic features for predicting hiv-1 drug resistance. Bioinformatics, 23(18):2455-2462.

Schölkopf, B. and Smola, A. J. (2002). Learning with kernels: support vector machines, regularization, optimization, and beyond. MIT press.

Uno, T., Kiyomi, M., and Arimura, H. (2005). Lcm ver.3: Collaboration of array, bitmap and prefix tree for frequent itemset mining. In Proceedings of the 1st International Workshop on Open Source Data Mining: Frequent Pattern Mining Implementations, OSDM '05, pages 77-86, New York, NY, USA. ACM.

Valiant, L. G. (1999). Projection learning. Machine Learning, 37(2):115-130.

Veropoulos, K., Campbell, C., and Cristianini, N. (1999). Controlling the sensitivity of support vector machines. In Proceedings of the international joint conference on AI, pages 55-60.

Wang, F. and Rudin, C. (2015). Falling rule lists. In Artificial Intelligence and Statistics, pages 1013-1022. Zhou, Z.-H., Jiang, Y., and Chen, S.-F. (2003). Extracting symbolic rules from trained neural network ensembles. Ai Communications, 16(1):3-15. 